\title{
Real-time dynamics of plasmonic resonances in nanoparticles described by a boundary elements method with generic dielectric function
}

\author{
Giulia Dall'Osto \\ Department of Chemical Sciences, University of Padova, via Marzolo 1, Padova, Italy \\ Gabriel Gil* \\ Department of Chemical Sciences, University of Padova, via Marzolo 1, Padova, Italy and \\ Instituto de Cibernetica, Matematica y Fisica, \\ Calle E esq 15 Vedado 10400, La Habana, Cuba \\ Silvio Pipolo \\ Université de Lille, CNRS, Centrale Lille, ENSCL, \\ Université d'Artois UMR 8181 Unité de Catalyse \\ et Chimie du Solide F-59000, Lille, France \\ Stefano Corni ${ }^{\dagger}$ \\ Department of Chemical Sciences, University of Padova, via Marzolo 1, Padova, Italy and \\ CNR Institute of Nanoscience, via Campi 213/A, Modena, Italy
}




\begin{abstract}
Investigating nanoplasmonics in an explicit time-dependent perspective is a natural choice when light pulses are used, and may also reveal aspects that are hidden in a frequency-based picture. In the past we proposed a method (TD-BEM) to simulate the time dependent polarization of nanoparticles based on a Boundary Element Method that is particularly suitable to interface with a quantum atomistic description of nearby molecules. So far, however, metal dielectric functions in TD-BEM have been modelled through analytic expressions, such as the Debye's and the DrudeLorentz's, which cannot account for multiple electronic resonances. Our approach allows to include in the TD BEM framework also the description of metals with complicate dielectric function profiles in the frequency domain. Particularly, among all metals, gold is a challenge case due to the presence of many transition frequencies. We applied our methods to different metals (gold, silver and the less commonly investigated rhodium) and different shaped NPs (spheres, ellipsoids and cubes), the approach has been tested comparing TD BEM and frequency domain BEM absorption spectra and it has been used to investigate the time-dependent field acting locally close to nanoparticles vertexes. .
\end{abstract}

\footnotetext{
* gabriel@icimaf.cu

† stefano.corni@unipd.it
} 


\section{INTRODUCTION}

Plasmon resonances of metal nanoparticles (NPs) allows to focus the electromagnetic radiation within the nanoscale,[1, 2] giving rise to the so called optical nanoatenna effect.[3] The localized field enhancement they provide gives rise to new phenomena such as surface enhanced Raman scattering, surface enhanced infrared absorption, and metal enhanced fluorescence.[1, 4, 5] Furthermore, when coupled to ultrafast lasers, plasmonic NPs might be key to disclose, probe and manipulate a host of other photochemical phenomena at a singlemolecule level, such as excited state dynamics, high-harmonic generation and photosynthetic energy-transfer pathways.[6-8]

From a theoretical viewpoint, localized surface plasmons and the associated electric field enhancement are in many circumstances (i.e., when quantum[9] and non-local effects[10] are not relevant) well described by classical electromagnetic modeling. The plasmonic material is described as a continuous medium characterized by an empirical frequency dependent dielectric function, and Maxwell equations are solved in the presence of the external electromagnetic field. In practice, several approaches are available to numerically solve Maxwell equations in the presence of dielectrics.[11] Among them, the Boundary Element Method (BEM) [12] has been successfully applied to investigated localized surface plasmons, first in the quasi-static (i.e., long wavelength) limit[13] and then for the full Maxwell equations[1416], providing accurate results for non trivial geometries.[17] The basic idea is to translate the differential Maxwell equations into integral equations defined on the boundaries of the nanostructure, where the proper boundary conditions of the fields/the potentials are imposed. The integral equations, that may be written in terms of fictitious surface current densities and charges[12], are then solved by proper discretization of the boundary into a mesh (boundary elements) leading to standard linear algebra problems.

BEM is usually formulated in the frequency domain (we call this Freq BEM, heretofore), i.e., monocromatic source EM fields have to be used. However, the time dimension is nowadays central in molecular plasmonics,[18] from the application of nanostructures together with laser pulses to achieve few-molecule sensitivity in femtochemistry measurements[19] to the exploration of chemical reactions in the strong coupling regime.[20] If results in the time domain are needed, a possible strategy (possible because of the linear character of macroscopic Maxwell equations) is to calculate the response for a large enough range of frequencies 
with a dense enough frequency grid, perform all the Freq BEM calculations and then derive the time-dependent physical quantities through a Fourier transform (FT). This is a viable but somewhat involved approach, that becomes unpractical when the dynamics of the localized surface plasmons is coupled with other dynamics, such as the electronic dynamics of a nearby molecule.[21] Beside switching to a wholly different numerical approach, such as Finite Difference Time Domain (FDTD)[11], an alternative way of getting to the time dependent description, is reformulating BEM in terms of equations of motion (EOM) governing the evolution of the relevant BEM quantities (we will call this TD BEM). Quasi-static TD BEM admits the real-time numerical propagation of polarization charges, one time step at a time.[21]

So far, the computational strategy tackling TD BEM has been accessible only for NP materials described by analytical frequency dependent dielectric functions, using either the Debye or the Drude-Lorentz (DL) form.[21, 22] Stemming from a free-electron treatment of the metal conduction, the DL model is adequate but it does not fully conform with the dielectric properties of realistic metallic NP media.[23] The main drawback of the DL model is that it includes a single electronic transition, while real metal involves free-electron behaviors as well as (in general) multiple interband transitions.

The main goal with this contribution is to lay down an extension of TD BEM to study plasmonic NPs featuring any general and physically sound dielectric function. Our proposal relies only on the knowledge of discrete values of the frequency-dependent, complex dielectric function $\epsilon(\omega)$, e.g., coming from measurements or ab initio calculations. The core idea is to fit an auxiliary function $f(\omega)$ (whose form is derived here) to a series of DL terms that respect key properties of a dielectric function, in turn inherited by $f(\omega)$. Similar fits to a DL expansion have been proposed before in the context of FDTD approaches.[23, 24] In particular, Ref.23 shows that a DL expansion with four terms accurately describe the dielectric function of challenging noble metals, such as gold.

The aforementioned DL series is convenient to derive TD BEM from Freq BEM equations. The result of such a derivation is a set of EOM (second-order differential equations representing a set of forced, damped and coupled harmonic oscillators) for BEM polarization charges, each associated with a different DL term in the expansion.

Based on such a description, we can accurately calculate the real-time dynamics of the electromagnetic field acting locally in a region of space nearby the plasmonic NP, once a 
laser pulse arbitrarily shaped in time impinges on the nanoparticle. Exploiting the relatively small number of elements coming from BEM compared to spatial formulation such as FDTD, this approach also opens up the way to effectively simulate the evolution of a molecular wavefunction described by quantum chemistry time dependent methods mutually coupled with a NP described by a generic dielectric function. To illustrate the numerical approach, we carry out real-time simulations on prototypical NPs made of different materials (gold, silver and rhodium) and shaped in different geometries (sphere, ellipsoid, cube and rod).

The outline of the article is as follows. In Sec. II, we formulate TD BEM starting from traditional Freq BEM in the quasi-static limit. Sec. III reports on our results, and is subdivided in various subsections. Sec. III B focus on the assessment of TD vs. Freq BEM for a prototypical case of a gold nanosphere. Sec. IIIC is devoted to a comparison of TD BEM against analytical results available for the absorption spectrum of specific NP geometries, such as a sphere and an ellipsoid. In Sec. III D, we perform further TD BEM tests in the case of silver and rhodium NPs with less symmetric shapes, such as a cube and a rod. Sec. III E shows the computational time scaling of TD and Freq BEM with respect to the number of tesserae in the NP surface discretization. In Sec. III F, we analyze the local time-dependent electric field near the NP (in particular, close to a gold nanocube corner). In Sec. III G we perform dynamics of an $\mathrm{LiCN}$ molecule close to a gold nanocube interacting with an incident electric field. Finally, Conclusions are given in Sec. IV.

\section{METHODS}

We treat the metal NP as a polarizable continuum body within the quasi-static approximation, i.e., neglecting retardation effects. In this framework, the NP is described through PCM-NP, [25] an extension of the Polarizable Continuum Model (PCM)[26] in the Integral Equation Formalism (IEF).[27] As it is customary with PCM, the problem is reformulated in terms of BEM for its numerical implementation: the NP surface is discretized in small portions, or tesserae, each of which is associated to an apparent charge located in its geometrical centre. The apparent surface charges enclose all the relevant electromagnetic information related to the interaction of the NP with an external potential due to, for example, an in-

cident electric field or the presence of a charge distribution (e.g., a molecule) nearby. The frequency dependent IEF-PCM equation of polarization charges associated to the tesserae 
is written as

$$
\mathbf{q}(\omega)=\mathbf{Q}(\omega) \mathbf{V}(\omega)
$$

where

$$
\mathbf{Q}(\omega)=-\mathbf{S}^{-1}\left(2 \pi \frac{\epsilon(\omega)+1}{\epsilon(\omega)-1} \mathbf{I}+\mathbf{D A}\right)^{-1}(2 \pi \mathbf{I}+\mathbf{D A}) .
$$

$\mathbf{D}$ and $\mathbf{S}$ are matrices representative of the Calderon's projectors whose out-of-diagonal matrix elements are given as $D_{i j}=\frac{\left(\vec{s}_{i}-\vec{s}_{j}\right) \cdot \vec{n}_{j}}{\left|\vec{s}_{i}-\vec{s}_{j}\right|^{3}}, S_{i j}=\frac{1}{\left|\vec{s}_{i}-\vec{s}_{j}\right|}$ and diagonal elements are $D_{i i}=$ $-\left(2 \pi+\sum_{k \neq i} D_{i k} A_{k}\right) \frac{1}{A_{i}}$ and $S_{i i}=1.0694 \sqrt{\frac{4 \pi}{A_{i}}},[26] \mathbf{A}$ is a diagonal matrix with elements equal to the area of the tesserae, $\epsilon(\omega)$ is the dielectric function of the metal and $\mathbf{V}(\omega)$ is the external frequency-dependent electrostatic potential associated, in this case, to an incident electromagnetic radiation in the long-wavelength limit. Here, we focus on the case of an incoming light exciting the NP, but actually our TD BEM treatment is general enough to tackle other situations, for instance, where the external potential is generated by a molecule close to the NP.

Equation 1 is rearranged to isolate the contribution that depends on the dielectric function, i.e.,

$$
\mathbf{q}(\omega)=\frac{1}{2 \pi} f(\omega) \mathbf{F}(\omega)
$$

with

$$
f(\omega)=\frac{\epsilon(\omega)-1}{\epsilon(\omega)+1}
$$

and

$$
\mathbf{F}(\omega)=-\left[\mathbf{A D}^{*} \mathbf{q}(\omega)+\mathbf{S}^{-1}(2 \pi \mathbf{I}+\mathbf{D A}) \mathbf{V}(\omega)\right]
$$

$f(\omega)$ is a function collecting all dependencies on $\epsilon(\omega)$, whereas $\mathbf{F}(\omega)$ builds up from the scalar potential of the impinging radiation field and a self-consistent dependence on polarization charges.

This equation is conceptually different from the standard formulation of IEF-PCM in eq. 1. While the physical meaning of eq.(1) is clear (q are the charges induced by the external electrostatic potential collected in $\mathbf{V}$ ), eq.(3) requires a specific discussion. The physical 
dimension of the term $\mathbf{F}(\omega)$ in eq. (3) is that of an area times an electric field, as shown by its first term and the definition of $\mathbf{D}$. Letting aside the area (that could be moved on the 1.h.s. obtaining surface charge density instead of charges), $\mathbf{F}(\omega)$ can be interpreted as the normal component of the total electric field acting in a given portion (tessera) of the surface. The factor $f(\omega)$ expresses the response of the dielectric medium to this total electric field, driving the local surface charge oscillation. In fact, the expression for $f(\omega)$ appears in other dielectric responses of a planar surface to an external perturbation, such as in the image potential for an external charge or dipole, and the zero of the denominator would correspond to the surface plasmon resonance of a planar surface. In equation 3 the total electric field depends on two contribution: the field produced by the charge distribution on the NP surface itself (first term of equation 5) and the external field (second term of equation 5). The mutual dependence of the polarization charges is representative of the macroscopic polarization, and it encodes the effect of the shape on the frequency of the nanoparticle resonances. In this perspective, eq.(3) indicates that the resonances of the nanoparticle as a whole can be interpreted as arising from the hybridization of the resonances of the single planar tesserae (all happening at the same frequency determined by the factor $f(\omega)$ ), with a small correction embodied in the diagonal element of the matrix $D$, due to the tesserae curvature coupled by the mutual electromagnetic interactions, and excitable by an external field.

Shifting to the time domain, equation 3 reads

$$
\mathbf{q}(t)=\frac{1}{2 \pi} \int_{-\infty}^{+\infty} d t^{\prime} f\left(t-t^{\prime}\right) \mathbf{F}\left(t^{\prime}\right)
$$

where $f(\tau)\left(\tau=t-t^{\prime}\right)$ and $\mathbf{F}\left(t^{\prime}\right)$ are the FT of $f(\omega)$ and $\mathbf{F}(\omega)$, respectively. The polarization charges in time domain depends on the whole history of the system through the integral as in equation 6. This equation is the starting point of our derivation, in a similar spirit of what was done starting from the equation 1 for molecules in solution described by a Debye dielectric function[22] and close to a NP, described by a DL expression.[21]

Compared to such previous works, we anticipate that the rearrangement leading to eq. 3 from eq. 1 has two important advantages, that will be illustrated in the rest of the work. They both derive from the possibility of writing the charges as a scalar function of the dielectric function $(f(\omega))$ times a vectorial term independent from the dielectric function. The first advantage is that the EOMs that we shall derive from eq. 1 do not require a preliminary 
matrix diagonalization, at odds with refs.[21, 22]. The second advantage lies particularly on the shape of $f(\omega)$ drawn from the dielectric function of choice. Previously,[21] the dielectric function of metals have been modelled by a DL expression. This approximation produce acceptable results in some cases but does not allow to properly describe metals with interband transitions. Among different metals, the description of gold plasmonic behavior is quite a numerical challenge due to the very complicated $\epsilon(\omega)$ profile, resulting from many transition frequencies. The strategy recently employed in the framework of FDTD to overcome this limitation is based on a formulation of the dielectric function as a sum of DL terms.[23, 24] However, we cannot apply directly the latter scheme, since the FT of $f(\omega)$, when substituting the so formulated expression for $\epsilon(\omega)$, is not useful to derive EOMs for the charges.

Our TD BEM approach overcomes such a difficulty by setting up a frequency-dependent model directly for $f(\omega)$ that (a) works for any dielectric function, (b) accounts for a collection of plasmonic frequencies, and (c) leads to a straightforward formulation in the time domain through EOMs. We fit $f(\omega)$ (built from an $\epsilon(\omega)$ known by points) to a sum over $N$ DL like terms, i.e.,

$$
f(\omega)=\frac{\epsilon(\omega)-1}{\epsilon(\omega)+1} \simeq \sum_{p=1}^{N} \frac{A_{p}}{\omega_{p}^{2}-\omega^{2}-i \gamma_{p} \omega} .
$$

This choice is motivated by the fact that $f(\omega)$ and $\epsilon(\omega)$ share a similar behaviour. Especially, both of them have poles lying on the lower half of the complex frequency plane, although $f(\omega)$ poles are $\epsilon(\omega)+1$ roots. Furthermore when $\epsilon(\omega)$ is written by a single DL term, we obtain again a DL expression for $f(\omega)$. As stated above, the term $f(\omega)$ represents the resonance frequencies of an infinite plane surface in the quasi-static limit. The shape chosen for the function $f(\omega)$ allows to include a number of resonances that, combined, generate the overall plasmonic resonance of the system. The number of poles of the function $f(\omega)$ determines the number of DL terms needed to obtain a reasonable accordance between its analytical expression and its numerical profile depending on the experimental dielectric function. Each DL term has two symmetric poles corresponding to the complex numbers

$$
\overline{\overline{\omega_{p}}}=-i \frac{\gamma_{p}}{2} \pm \sqrt{\omega_{p}^{2}-\frac{\gamma_{p}^{2}}{4}}=-i \frac{\gamma_{p}}{2} \pm \overline{\omega_{p}} .
$$

Practically, we perform the regression in two steps. First, the imaginary part of $f(\omega)$ is 
fitted to $N-1$ DL terms, and then, an extra DL term with real poles $\left(\gamma_{p}=0\right)$ is added to fit the real part of $f(\omega)$. This last function accounts for a vertical translation of the real part of $f(\omega)$, i.e., it takes care of the high frequency behaviour of the dielectric function that, in the $\mathrm{UV} /$ visible spectral region, gives rise to an approximately constant real part of $\epsilon(\omega)$.

Introducing the fitted function $f(\omega)$ in equation 3 , the polarization charges can be decomposed as a sum of pole-dependent charges $\mathbf{q}_{p}(\omega)$, i.e.,

$$
\mathbf{q}(\omega)=\sum_{p}^{N} \mathbf{q}_{p}(\omega)
$$

where

$$
\mathbf{q}_{p}(\omega)=\frac{1}{2 \pi} \frac{A_{p}}{\omega_{p}^{2}-\omega^{2}-i \gamma_{p} \omega} \mathbf{F}(\omega) .
$$

Note that each pole-dependent charge is affected by the rest of the charges through a recursive dependence on the total charge $\mathbf{q}(\omega)$.

A simple way to obtain the EOM is provided by direct FT of the pole-dependent charges in eq.(10) written in a compact form, i.e.,

$$
\left(\omega_{p}^{2}-\omega^{2}-i \gamma_{p} \omega\right) \mathbf{q}_{p}(\omega)=\frac{A_{p}}{2 \pi} \mathbf{F}(\omega)
$$

Recalling in particular that

$$
\begin{aligned}
& \ddot{\mathbf{q}}_{p}(t)=-\frac{1}{2 \pi} \int_{-\infty}^{+\infty} d \omega e^{-i \omega t} \omega^{2} \mathbf{q}_{p}(\omega), \\
& \dot{\mathbf{q}}_{p}(t)=-i \frac{1}{2 \pi} \int_{-\infty}^{+\infty} d \omega e^{-i \omega t} \omega \mathbf{q}_{p}(\omega),
\end{aligned}
$$

we got a second order differential equation, i.e.

$$
\ddot{\mathbf{q}}_{p}(t)=-\omega_{p}^{2} \mathbf{q}_{p}(t)-\gamma_{p} \dot{\mathbf{q}}_{p}(t)+\frac{A_{p}}{2 \pi} \mathbf{F}(t)
$$

where

$$
\mathbf{F}(t)=-\mathbf{A D}^{*} \mathbf{q}(t)-\mathbf{S}^{-\mathbf{1}}(2 \pi \mathbf{I}+\mathbf{D A}) \mathbf{V}(t)
$$

All the geometrical features of the NP are included in $\mathbf{F}(t)$. Equation 14 resembles the one for a set of coupled forced and damped harmonic oscillators (each characterized by a displacement $q_{p, i}$ where $i$ is the tessera index), where $\sqrt{\omega_{p}^{2}+\frac{A_{p}}{2 \pi} \mathrm{A}_{i i} D_{i i}^{*}}$ is the intrinsic 
resonance frequency of the $q_{p, i}$ oscillator, that is then coupled to all the other $q_{p^{\prime}, j}$ oscillators via the $\mathrm{A}_{i i} D_{i j}^{*} q_{p^{\prime}, j}$ terms. The set of EOM 14 (with $p=1, \ldots, N$ ) extends the analogous result in Ref. [21] (derived for a dielectric function with a single DL term) to the case of several resonance frequencies. At the same time it is a general method able to describe different metal NPs, once we know $\epsilon(\omega)$.

Practically, we integrate equation 14 by means of the velocity-Verlet algorithm.[28] In particular, we get

$$
\mathbf{q}_{p}(t+d t)=\mathbf{q}_{p}(t)+\dot{\mathbf{q}}_{p}(t) d t+\left[-\omega_{p}^{2} \mathbf{q}_{p}(t)-\gamma_{p} \dot{\mathbf{q}}_{p}(t)+\frac{A_{p}}{2 \pi} \mathbf{F}(t)\right] \frac{d t^{2}}{2},
$$

and

$$
\begin{aligned}
\dot{\mathbf{q}}_{p}(t+d t)= & \dot{\mathbf{q}}_{p}(t)+\left[-\omega_{p}^{2} \mathbf{q}_{p}(t)-\omega_{p}^{2} \mathbf{q}_{p}(t+d t)+\frac{A_{p}}{2 \pi}(\mathbf{F}(t)+\mathbf{F}(t+d t))\right] \frac{d t}{2} \\
& -\gamma_{p} \dot{\mathbf{q}}_{p}(t) d t+\left[\gamma_{p}^{2} \dot{\mathbf{q}}_{p}(t)+\gamma_{p} \omega_{p}^{2} \mathbf{q}_{p}(t)-\gamma_{p} \frac{A_{p}}{2 \pi} \mathbf{F}(t)\right] \frac{d t^{2}}{2} .
\end{aligned}
$$

The EOM of pole-dependent charges $\mathbf{q}_{p}(\omega)$ due to the last purely real term in $f(\omega)$ expansion would be even simpler since the imaginary part of the pole is missing. In the analyzed cases, this pole generates in time domain, a non-decaying, faster and less ample oscillations in comparison with other contributions to the total polarization charge. The presence of this term is crucial for the choice of the time step, which needs to be very small to correctly describe its fast oscillation. However, the equation in frequency domain can be simplified as

$$
\mathbf{q}_{p}(\omega)=\frac{1}{2 \pi} \frac{A_{p}}{\omega_{p}^{2}-\omega^{2}} \mathbf{F}(\omega) \simeq \frac{1}{2 \pi} \frac{A_{p}}{\omega_{p}^{2}} \mathbf{F}(\omega)
$$

within the limit of $\omega_{p}^{2} \gg \omega^{2}$, which is verified in the spectral range where common dielectric functions are experimentally available. Therefore, the EOM for the last pole reads

$$
\mathbf{q}_{p}(t)=\frac{1}{2 \pi} \frac{A_{p}}{\omega_{p}^{2}} \mathbf{F}(t)
$$

A first order finite-difference approximation to eq. 19 reads

$$
\mathbf{q}_{p}(t+d t)=\mathbf{q}_{p}(t)+\frac{1}{2 \pi} \frac{A_{p}}{\omega_{p}^{2}}(\mathbf{F}(t+d t)-\mathbf{F}(t))
$$


Otherwise the term $\mathbf{q}_{p}(t)$ can be made explicit from the sum over pole-dependent charges in $\mathbf{F}(t)$ as

$$
\mathbf{q}_{p}(t)=\left(1-\frac{1}{2 \pi} \frac{A_{p}}{\omega_{p}^{2}} \mathbf{A} \mathbf{D}^{*}\right)^{-1} \frac{1}{2 \pi} \frac{A_{p}}{\omega_{p}^{2}}\left[\mathbf{A} \mathbf{D}^{*} \sum_{k}^{N-1} \mathbf{q}_{k}(t)+\mathbf{S}^{-1}(2 \pi \mathbf{I}+\mathbf{D A}) \mathbf{V}(t)\right]
$$

Equations 20 and 21 give the same results in the $d t \rightarrow 0$ limit and both of them have been implemented, however the latter requires a matrix inversion that increase the computational cost of the calculation so we use the first method (equation 20) as default. It has to be noticed that the velocity-Verlet algorithm is a second-order accurate integrator, while propagator in equations 20 has a first order accuracy. Thus, Eq. 20 can lead to less precise results when the contribution of this last term becomes large in $\mathbf{q}(t)$. Equation 21, on the other hand, comes about from solving exactly for $\mathbf{q}_{p}$ in equation 19 (even though equation 19 itself is an accurate approximation of equation 14).

Another strategy to obtain the equation of the polarization charges dependent on the last pole consist in taking the second derivative of equation 19

$$
\ddot{\mathbf{q}}_{p}(t)=\frac{1}{2 \pi} \frac{A_{p}}{\omega_{p}^{2}} \ddot{\mathbf{F}}(t)
$$

The equation of motion for the last pole is obtained through Velocity-Verlet propagator, a second-order accurate algorithm

$$
\begin{aligned}
& \mathbf{q}_{p}(t+d t)=\mathbf{q}_{p}(t)+d t \dot{\mathbf{q}}_{p}(t)+\frac{A_{p}}{2 \omega_{0 p}^{2} \pi} \ddot{\mathbf{F}}(t) \frac{d t^{2}}{2} \\
& \dot{\mathbf{q}}_{p}(t+d t)=\dot{\mathbf{q}}_{p}(t)+\frac{A_{p}}{2 \omega_{0 p}^{2} \pi}(\ddot{\mathbf{F}}(t+d t)+\ddot{\mathbf{F}}(t)) \frac{d t}{2}
\end{aligned}
$$

where

$$
\ddot{\mathbf{F}}(t)=\frac{1}{d t^{2}}[3 \mathbf{F}(t-d t)-8 \mathbf{F}(t-2 d t)+7 \mathbf{F}(t-3 d t)-2 \mathbf{F}(t-4 d t)]
$$

Equation 23 is computationally more demanding with respect to equations 20 and 21 because we need to store a larger number of data for each cycle since $\ddot{\mathbf{F}}(t)$ at the second order accuracy depends on $\mathbf{F}(t)$ at 4 different time steps. On the other hand this algorithm is second order accurate (same accuracy of the Velocity-Verlet algorithm employed for propagation of the other pole-dependent charges) and for this reason it has been employed when previous approaches need very short time steps to give accurate enough results. 


\section{RESULTS AND DISCUSSION}

This section reports on real-time simulations of the NP polarization charges induced by an incident electromagnetic field. In order to assess our TD BEM approach, the results from time-dependent propagation of polarization charges are compared with those stemming from direct BEM calculation in the frequency domain (i.e., Freq BEM), as in Ref.21. The quantity we check when testing TD BEM accuracy and performance is the NP isotropic polarizability $\alpha(\omega)$, which is linked to the absorption cross section through the linear equation[29]

$$
\kappa_{\alpha}=\frac{4 \pi \omega}{n(\omega) c} \operatorname{Im}\{\alpha(\omega)\}
$$

where $n(\omega)$ is the refractive index of the medium surrounding the NP, here assumed to be vacuum. The isotropic polarizability is computed as

$$
\begin{aligned}
& \alpha(\omega)=\frac{1}{3} \sum_{i=1}^{3} \frac{d_{i}(\omega)}{E_{i}(\omega)} \\
& \alpha(\omega)=\frac{1}{3} \sum_{i=1}^{3} \frac{1}{E_{i}(\omega)} \int_{-\infty}^{\infty} d t d_{i}(t) e^{i \omega t},
\end{aligned}
$$

in the case of Freq and TD BEM, respectively. $E_{i}(\omega), d_{i}(\omega)$ and $d_{i}(t)$ are the $i$-th Cartesian components of the external electric field, the NP dipole moment in the frequency domain and its FT to time domain.

\section{A. Computational details}

Within all of our TD simulations, the dynamics is $32 \mathrm{fs}$ long, and the incident electric field features a gaussian dependence in time and a linear polarization along the x-axis. We assign different time steps depending on the modelled situation. In most cases and unless stated otherwise, such an electric field is written as

$$
\vec{E}(t)=\vec{E}_{0} e^{-\frac{t^{2}}{2 \sigma^{2}}}
$$

where the intensity $I=\frac{1}{2} \epsilon_{0} c\left|E_{0}\right|^{2}=10^{6} \mathrm{~W} / \mathrm{cm}^{2}$. This electric field shape is close to a delta pulse, relative to the propagation time scale, and thus it can excite a broad band at optical wavelengths. 
All the calculations have been performed on an Intel Xeon 24 Core with 256 GB of RAM and 2 x 300 GB HD SAS (10 krpm). Both TD BEM and Freq BEM fortran 90 codes have been compiled with the ifort compiler and optimized with the compilation FLAGS: -O3 -ftz -align all -heap-arrays 1024. We employed the software gmsh to produce 2D meshes of the NPs surfaces that generates triangular and quadrangular finite elements. Charges propagation have been performed through TDPlas, a homemade code.

The fitting of the function $f(\omega)$ obtained by the experimental dielectric function of the metals considered here (gold[30], silver [31] and rhodium [32]) to a sum of DL like terms plus the high-frequency, purely real DL term discussed previously has been performed by an homemade Matlab script. In the first place, we have to fix the number of DL terms in the fitting function. There is a clear trade-off between optimal fit quality and computational time. Increasing the number of parameters, which in our scheme means to increase the number of DL terms in equation 7, can accomplish systematically better regressions, but it also implies considering more (coupled) EOM to integrate (see equations 14, 16 and 17), slowing down the real-time simulations. As good compromises between these two aspects, we chose six (five plus the high frequency real term) terms for all the three metals considered here. Data and plots with different numbers of DL terms in the expansion of $f(\omega)$ for gold, silver and rhodium are reported in the SI.

In Sec. III G we report the dynamics of an $\mathrm{LiCN}$ molecule close to a gold nanocube performed with the homemade code WaveT.[21, 33] The molecule is described in terms of 10 electronic excited states (energies have been reported in table S11 of supplementary material) and relative transition dipole moments calculated at CIS level of theory. All the information related to the molecule and the potential on the NP tesserae due to the presence of the molecule have been computed with a locally modified version of Gamess.[34, 35]

\section{B. Comparison with frequency domain calculations results}

In order to assess our time propagation scheme to tackle a NP described by a general dielectric function, here we compare the results of TD BEM with a Freq BEM benchmark. The frequency dependent polarization charge on each tessera is calculated through equation 1.

Before analyzing the accuracy of TD BEM, we test approximation 7 by contrasting Freq 
BEM results with the fitted and the experimental dielectric function. Such a test allows to determine whether the fitted function is good enough for practical use.

In Fig. 1a, we report the fitting of the function $f(\omega)$ obtained by the experimental dielectric function of gold [30] to a sum of five DL like terms plus the high-frequency, purely real DL term discussed previously. We chose the smaller possible number of DL terms that lead to the best fitting of the function $f(\omega)$. Fitting parameters are reported in table I. Since $f(\omega)$ is effectively acting as a response function connecting the total electric field acting on a tessera and the induced charge density, the fitting parameters could be identified as the frequency, damping and strength of a series of excitations of the single tessera. However, the parameters $A_{p}$ obtained from the fitting assume both positive and negative values: in principle this result is nonphysical since $A_{p}$ should be squared terms of matrix elements[36] (or a generalized plasma frequency in a classical perspective)[37] and therefore positive. Nevertheless, the fitting of the function $f(\omega)$ for gold using only positive value of $A_{p}$ leads to inaccurate results even if more terms are included in the fitting (data not shown). The latter findings is in line with a discussion in the literature on the model required to best fit a metal dielectric functions when interband transitions are present, [38] that includes the possibility of using complex (and thus potentially real negative) $A_{p}$ and poles of higher order. Fitting of the silver dielectric functions with the model proposed in ref. 38 indeed provided $A_{p}$ with negative real parts.[39] In particular, we note that the values with the lowest values of $\omega_{p}$ comes in pair (i.e., $\omega_{p}=2.43 \mathrm{eV}, 2.49 \mathrm{eV}$ and $\omega_{p}=2.60 \mathrm{eV}, 2.64 \mathrm{eV}$ ) that

TABLE I. Parameters of $f(\omega)$ when substituting the gold dielectric function, fitted by six DL like terms.

\begin{tabular}{lccc}
\hline$p$ & $\omega_{p}$ & $\gamma_{p}$ & $A_{p}$ \\
$(\mathrm{eV})$ & $(\mathrm{eV})$ & $\left(\mathrm{eV}^{2}\right)$ \\
\hline 1 & 2.43 & 0.882 & -5.19 \\
2 & 2.49 & 0.287 & 0.675 \\
3 & 2.60 & 1.17 & 7.90 \\
4 & 2.64 & 4.53 & -15.4 \\
5 & 7.49 & 3.76 & 63.0 \\
6 & 42.4 & 0 & 643 \\
\hline
\end{tabular}



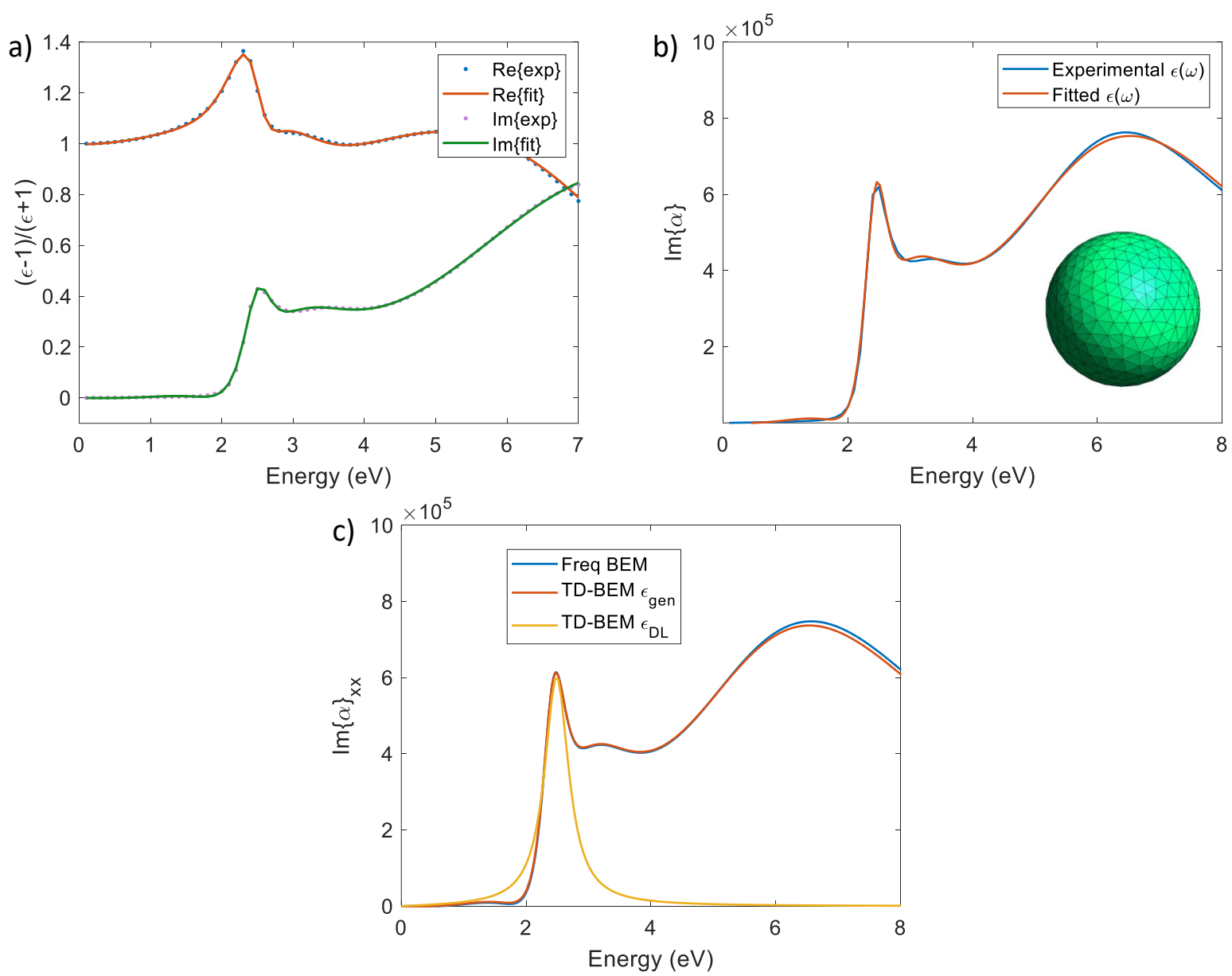

FIG. 1. a) Fitting of Au function $f(\omega)$ with a sum of six DL like equations, b) comparison between imaginary part of polarizability of Au nanosphere calculated with TD BEM and Freq BEM with experimental dielectric function in order to verify the reliability of the fitted equation $f(\omega)$ with respect to the experimental dielectric function c) comparison between imaginary part of polarizability of $\mathrm{Au}$ nanosphere calculated with Freq BEM and TD BEM employing dielectric function from fitted parameters and with TD BEM employing one Drude-Lorentz equation as dielectric function with resonance frequency closer to the peak at $2.3 \mathrm{eV}$.

effectively provide asymmetric line shapes in the range of frequency around the $\omega_{p}$.

We perform real-time simulations on a prototypical NP, a gold nanosphere with $5 \mathrm{~nm}$ radius, subject to the electric field in equation 28. Here, the time step is 2.42 as $(0.1 \mathrm{au})$ and the field duration is set by $\sigma=0.0242 \mathrm{fs}$. We use a sphere surface discretization with 352 boundary elements (tessere).

Fig. 1b shows the imaginary part of the polarizability resulting from Freq BEM calcula- 
tion with the experimental dielectric function and from TD BEM calculation with the fitted dielectric function. As expected, the fitting results match very well those obtained from the experimental dielectric function, in particular for the position and the height of the plasmon peak at $2.5 \mathrm{eV}$. Minor discrepancies can be seen for the interband peak around $3 \mathrm{eV}$. If needed, systematic improvement of the fitting is possible by increasing the number of DL terms.

In Fig. 1c, we report the imaginary part of the polarizability calculated by Freq and TD BEM, both featuring the fitted dielectric function and the polarizability obtained by TD BEM with the dielectric function modelled by the Drude-Lorentz equation whose parameters are chosen in order to represent the peak due to the transition frequency at $2.3 \mathrm{eV}$. The parameters employed to modelled the Drude-Lorentz dielectric function are $\omega_{p}=2.32 \mathrm{eV}$, $\gamma_{p}=0.464 \mathrm{eV}, A=2.61 \mathrm{eV}^{2}$. As apparent in the latter plot, methods TD BEM (with general dielectric function) and Freq BEM are in good agreement. The two profiles are quite superposed but for a slight displacement between 6 and $8 \mathrm{eV}$ that depends on the choice of the propagation time step. The numerical effect of the time step is discussed in the supplementary material. On the other hand, the results obtained with a dielectric function modelled through a Drude-Lorentz equation is very partial since it can take into account only one resonance frequency. In sight of these results, the time-propagation algorithm employed can be considered validated with respect to the same BEM formalism in frequency domain.

\section{Ellipsoidal and spherical NP: comparison with analytical results}

An analytical solution of the Poisson equation is available for simple homogeneous shapes such as spheres and ellipsoids.[40] The polarizability of a spherical NP is the following

$$
\alpha(\omega)=3 V \epsilon_{s} \frac{\epsilon(\omega)-\epsilon_{s}}{\epsilon(\omega)+2 \epsilon_{s}}
$$

where $V$ is the volume of the sphere, $\epsilon(\omega)$ is the dielectric function of the metal and $\epsilon_{s}$

is the dielectric constant of the surrounding medium. Analogously, the equation for the polarization along the dimension $j$ of an ellipsoidal NP is calculated as 
a)

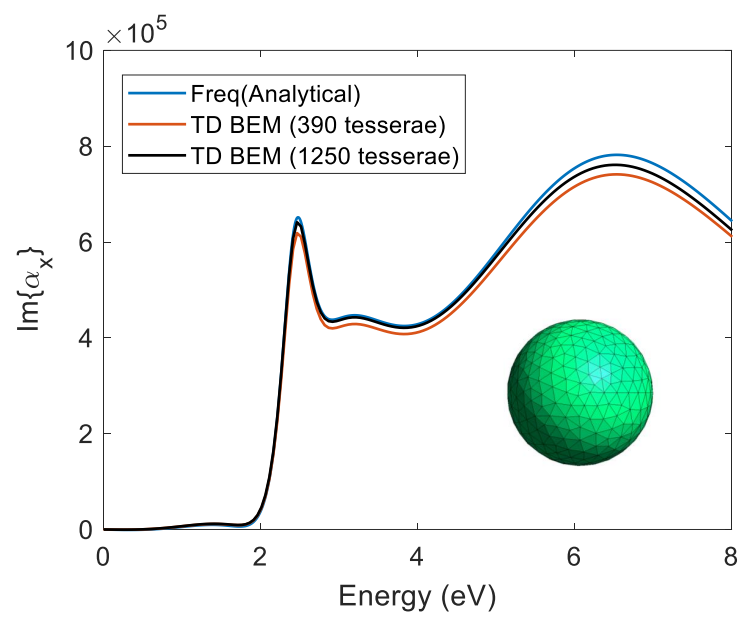

b)

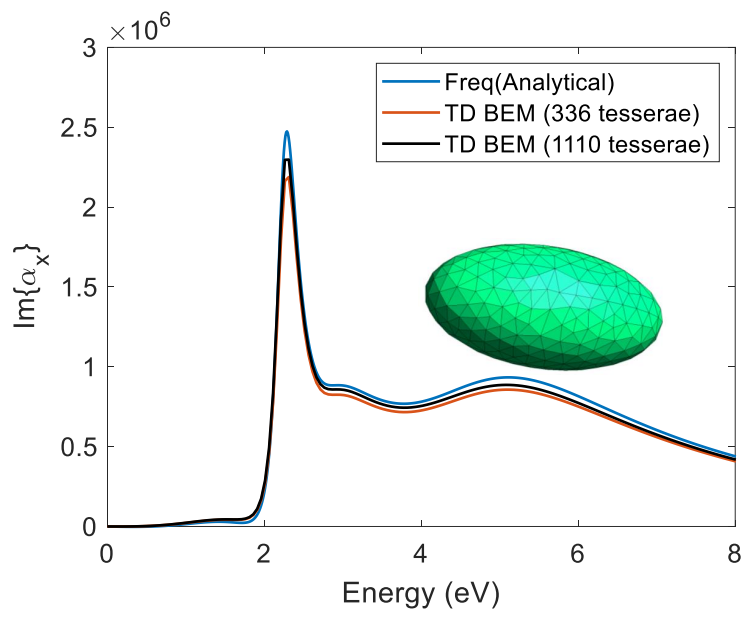

FIG. 2. Comparison between analytical calculation performed with eq. 29 and eq. 30 and TD BEM results obtained for a) Au nanosphere, b) Au nanoellipsoid polarized along $\mathrm{x}$ axis. The TD BEM calculations have been performed varying the number of tesserae.

$$
\begin{gathered}
\alpha_{j}(\omega)=V \epsilon_{s} \frac{\epsilon(\omega)-\epsilon_{s}}{\epsilon_{s}+L_{j}\left(\epsilon(\omega)+\epsilon_{s}\right)} \\
L_{j}=\frac{R_{1} R_{2} R_{3}}{2} \int_{0}^{\infty} \frac{d s}{\left(s+R_{j}^{2}\right)^{2} \sqrt{\left(s+R_{1}^{2}\right)^{2}+\left(s+R_{2}^{2}\right)^{2}+\left(s+R_{3}^{2}\right)^{2}}}
\end{gathered}
$$

where $R_{1}, R_{2}, R_{3}$ are the semiaxes lengths of the ellipsoid.

Figure 2 reports the comparison between equations 29 and 30 and TD BEM results obtained for a gold nanosphere with radius $r=5 \mathrm{~nm}$ (panel (a)) and a gold nanoellipsoid with semi-axes $R_{1}=10 \mathrm{~nm}$ and $R_{2}=R_{3}=5 \mathrm{~nm}$ (panel (b)). In both cases, the modelled NP is placed in vacuum $\left(\epsilon_{s}=1\right)$ and it is excited by a gaussian electric field with $\sigma=0.0242$ fs (see equation 28). We compute the polarizability along the direction of the electric field, which in the case of the ellipsoidal NP coincide with its major axis. Here, we consider two different resolutions of tessellation in order to study the effect of the number of tesserae on the final results. Spherical NP surface is divided in 390 and 1250 tesserae, while for the ellipsoidal one 336 and 1110 tesserae have been used. The results confirm the agreement between the analytical solutions of the Poisson problem and the TD BEM results. In addition, a better correspondence is found when a larger number of tesserae is employed, as expected, due to the effective volume of the discretized sphere (practically a polyhedron) that increase with the number of tesserae and tends to the volume of the sphere. The relative variation between 

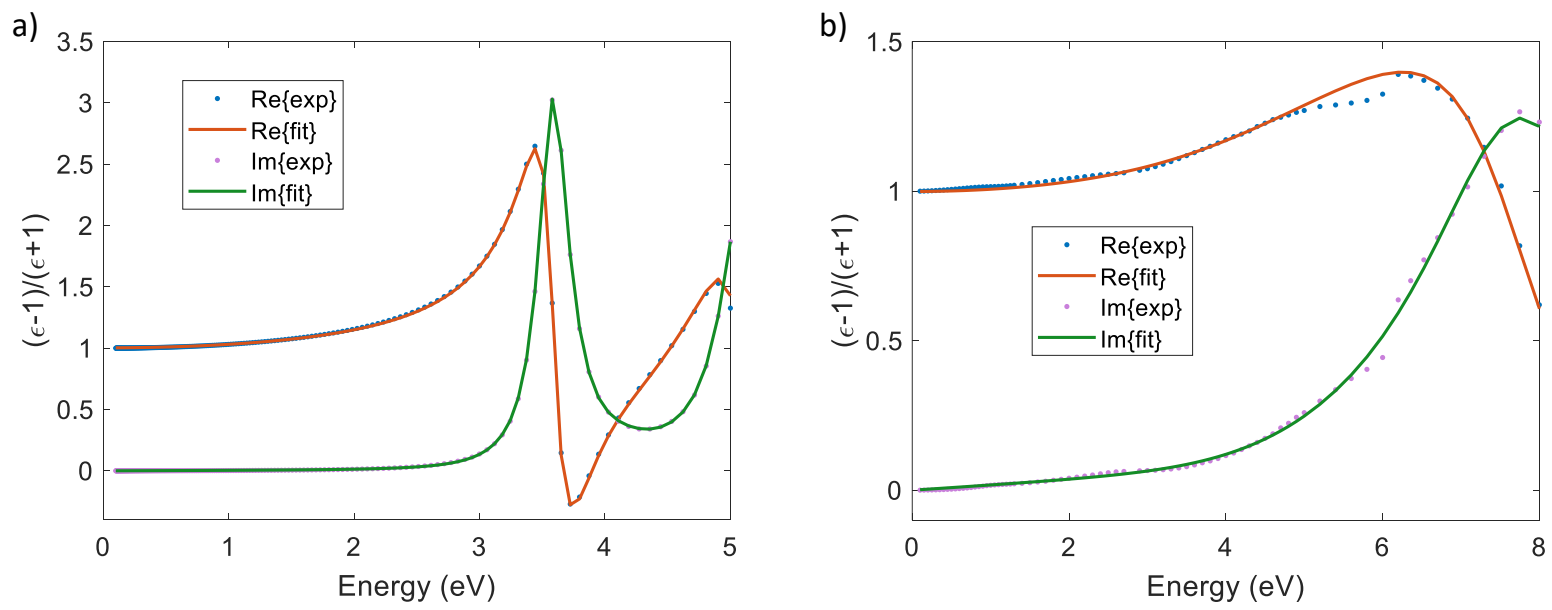

FIG. 3. a) Fitting of Ag function $f(\omega)$ with a sum of six DL like equations, b) Fitting of Rh function $f(\omega)$ with a sum of six DL like equations.

the profile of the imaginary part of polarizability with different tessellation resolution is larger for the spherical NP. As a consequence of the larger curvature of ellipsoidal NP, a larger resolution is needed to obtain the same calculation accuracy with respect to spherical NP.

\section{Different metals and different NP shapes}

Next, we perform other simulations varying the NP geometries and materials. Specifically, we consider cubes and rods shapes together with Ag and Rh metals, in all combinations. Silver and rhodium dielectric functions were obtained from Refs.31 and 32, respectively. Again, we fit $f(\omega)$ with a DL expansion with six terms, one of which misses the imaginary part. Figure 3 shows the plot of the fitted function $f(\omega)$ of silver and rhodium while the fitted parameters have been reported respectively in Table S6 and Table S10 of supplementary material. We use the data reported in ref. [32] for the dielectric function of rhodium: these data are collected from two different measurement that lead to the discrepancy around 6 $\mathrm{eV}$, because a single experimental measurement of dielectric function was not available in the region $0-8 \mathrm{eV}$.

In every TD BEM propagation, the $\mathrm{NP}$ is interacting with an electric field polarized along the larger dimension of the rod and normal to the cube face, in each case. Here, the bandwidth is $\sigma=0.0242$ fs (see equation 28) and the time step is $d t=2.42$ as.

We model the nanocube as a geometric cube with smooth edges and vertexes and the 

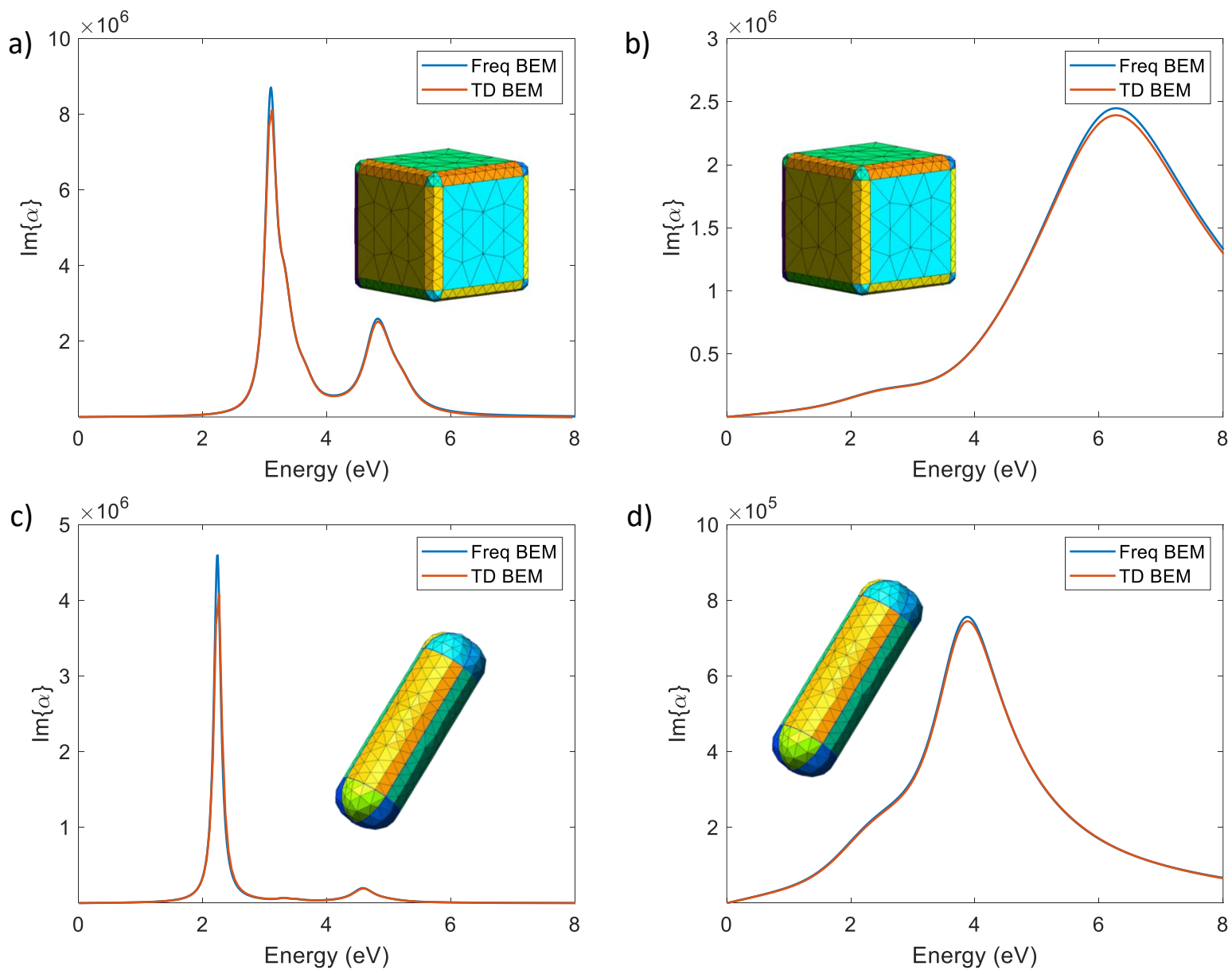

FIG. 4. Imaginary part of the polarizability of a) Ag nanocube, b) Rh nanocube, c) Ag nanorod, d) Rh nanorod calculated in frequency and time domain, within BEM formalism.

nanorod as a cylinder with same radius hemispheres covering both flat faces. In particular, the nanocube has a side length of $10 \mathrm{~nm}$ and a curvature radius at the edges of $1 \mathrm{~nm}$, whereas the nanorod is $10 \mathrm{~nm}$ long and has cylinder radius of $1.5 \mathrm{~nm}$. In both cases the surface is tasselled with 636 tesserae. In figure 4, we plot the polarizabilities of silver and rhodium nanocubes and nanorods.

In all the reported cases, the last pole-dependent charges are propagated through equation 23 instead of equation 20, employed in previous sections for gold NPs, in order to obtain results in full agreement with the Freq BEM simulated spectra. The latter algorithm (eq. $20)$ is first order accurate and thus it is more sensitive on time step variation. Particularly when simulating silver and rhodium NPs, a smaller time step (0.242 as) is needed to obtain good results. The polarizability of silver nanocube and nanorod shows very narrow peaks 
(therefore sensitive to the long-time behavior of the charge oscillations) that are not fully reproducible, in term of intensity, with a first-order accurate algorithm and the same time step employed with a second-order accurate approach. On the other hand, the necessity of a more accurate algorithm for simulating rhodium NPs is related to the parameters of the function $f(\omega)$ obtained from the fitting to the experimental values of $f(\omega)$ for rhodium. The real term of the equation 7 has a weight, $A_{p}$ larger than in gold or silver fitting, such that it influences the spectra at optical frequencies in a stronger way.

Silver and rhodium, compared to gold, have almost twice as large a ratio $A_{p} / \omega_{p}^{2}$ for the high frequency real term, which is the factor multiplying the external perturbation in eq.19. The associated $\mathbf{q}_{p}$ set of charges is therefore more relevant for silver and rhodium, and a better description of such term (i.e., second order vs first order) is therefore needed. Through equation 23, a second-order accurate algorithm, the propagation is performed with a longer time step of 2.42 as and consequently with a shorter calculation time.

The results of time dependent calculations have been compared with the results of the frequency formulation of BEM where the dielectric function is obtained from the fitted parameters (which anyway matches well the spectra obtained by the experimental dielectric functions). Freq and TD BEM simulated spectra are fully compatible. The minor discrepancies seen for the plasmonic peak of silver is due more to the discretization of the spectrum in the frequency domain than on real differences between the two approaches.

\section{E. Computational scaling of frequency and time domain calculations}

Our main interest in TD BEM is in the extra information on the nanoplasmonic system it straightforwardly provides, in terms of the time evolution of the local field and the real-time representation of the surface charge density. Yet, it should also have a better scaling of the computational time with number of tesserae compared to the same calculation performed in the frequency domain. Indeed, the algorithm employed for the charge propagation in time domain scales approximately as the square of the number of tesserae, since the most computationally demanding calculation is a vector-matrix product (see equation 16 and 17). On the other hand, BEM in frequency domain includes also matrix inversions (or matrix diagonalization, depending on the implementation) and matrix-matrix products that leads to an overall computational time scaling (formally) as the third power of the number of tesserae. 


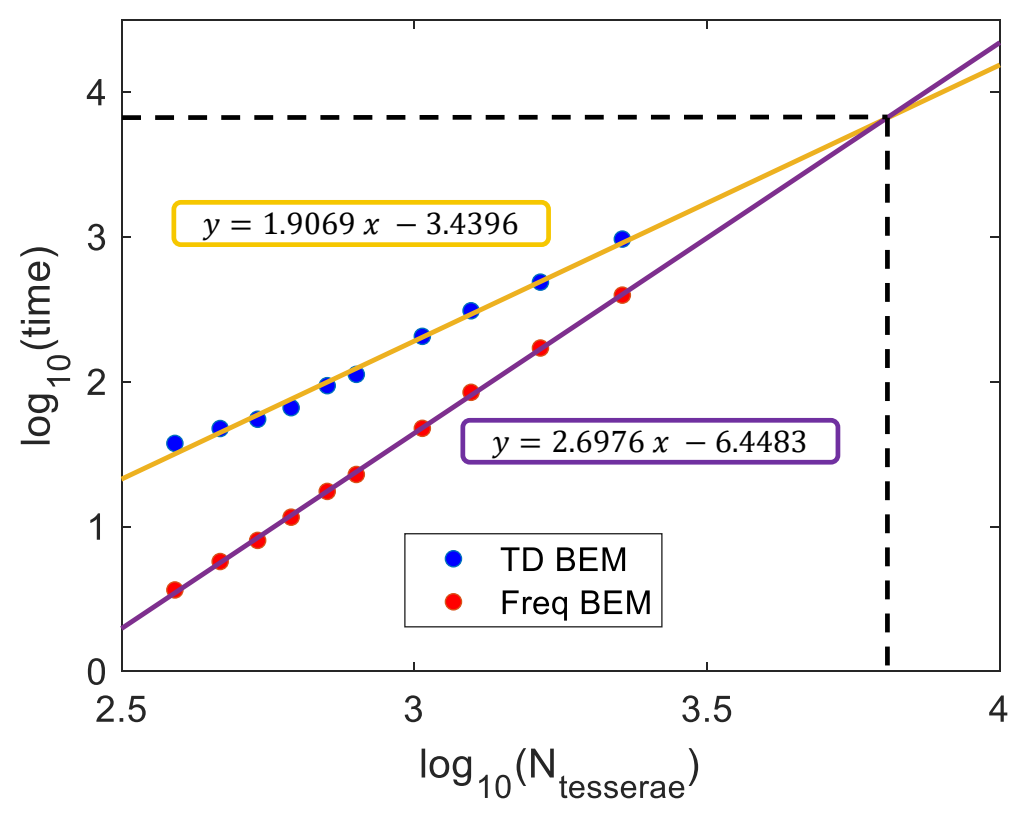

FIG. 5. Time calculation as a function of the number of tesserae employed for the NP surface tessellation in a double decimal logarithmic scale, for calculation in time and frequency domain. The dashed lines indicate where TD BEM becomes more convenient than Freq BEM for the calculation of absorption spectra in our implementations. Computational time is in seconds.

Therefore, for a large enough number of tesserae TD BEM should be more computationally inexpensive than Freq BEM.

In Fig. 5, we show the different computational time scaling of TD and Freq BEM runs for different tessellation resolutions, from 390 up to 2266 tesserae. Each point in the graph corresponds to a full polarizability spectrum calculation of a gold nanosphere with radius $r=5 \mathrm{~nm}$, taken as a representative case. The spectral region we want to explore is fixed between $0 \mathrm{eV}$ and $10 \mathrm{eV}$, with an energy resolution of $0.01 \mathrm{eV}$. TD BEM simulations are performed with a $d t=2.42$ as time step and are $32 \mathrm{fs}$ long, meaning $1.3 \times 10^{4}$ propagation steps. Actually such time step would allow a much larger spectral region to be explored, that would require more calculations in the frequency domain compared to what we performed. Yet, such ample spectral regions are usually not of interest (and would require additional fitting of the dielectric function), so we do not push the comparison to what is technically possible with TD BEM and stay rather with what is useful for calculations of the absorption spectra. 
The double logarithmic plot shows that both TD BEM and Freq BEM computational times stay on straight lines (i.e., they have polynomial scaling as expected). The slope of the straight line (i.e., the exponent of the scaling fitting the computational time) in TD BEM calculations is close to 2 while the data collected from frequency domain calculation lies on a line with slope around 2.7 (instead of 3 ). These numerical results substantially confirm the theoretical expectations. In practice, employing a relatively small number of tesserae, the algorithm developed in frequency domain is more convenient than that in time domain in terms of computational cost. When the number of tesserae increases, the situation is the other way around, being the time domain algorithm the most efficient. Notice that the overturn takes place near 3800 tesserae. Considering small aggregates of only 2 NPs and the most accurate tessellation resolution we have employed thus far, TD BEM calculations will be already more convenient than Freq BEM. The time scaling of Freq BEM and TD BEM approaches clearly depends on their code implementation. Particularly the Freq BEM code has been optimized through a diagonal formulation of IEF-PCM equation in which the calculation is performed through an initial slow step (the diagonalization of the $\mathbf{Q}(\omega)$ matrix in eq. 1, that formally scales as $N_{\text {tesserae }}^{3}$ ) followed by faster steps (vector-matrix products). There are different possible implementation of the same approach that will lead to different overall computational performances. Here, our purpose is to check that the computational scaling of the present implementation of Freq BEM and TD BEM as a function of the number of tesserae, conforms to the theoretical expectations.

\section{F. Local electric field nearby the NP.}

As stated in the Introduction, the main goal of the present approach is to provide a viable strategy to accurately investigate time-dependent fields in proximity of the metal nanoparticles. Here, we perform simulations on a gold nanocube (geometrically identical to those of Sec. IIID) interacting with a laser pulse described as a sinusoidal electric field modulated by a gaussian envelope. We are interested in illustrating the relation between the pole complex frequency and properties of the local field induced by the incidence of an external electric field, which is tuned to include a plasmonic resonance frequency within its spectral shape. The applied field evolve in time as 


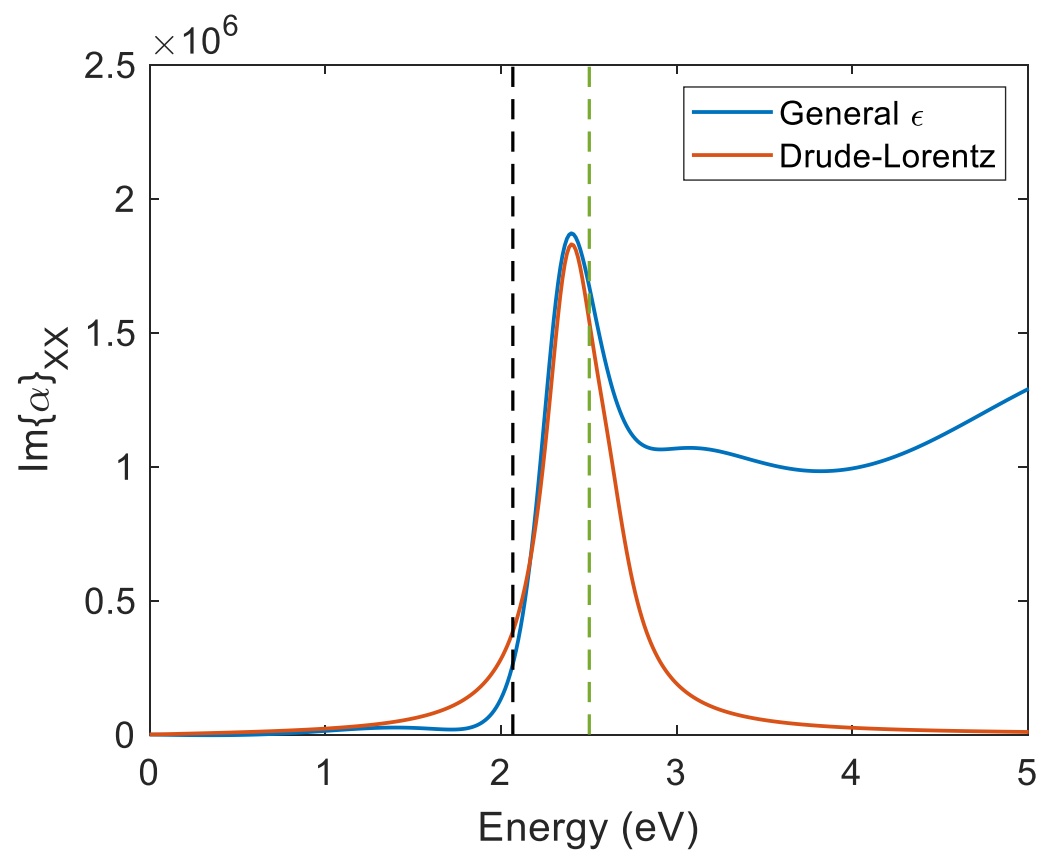

FIG. 6. Absorption spectrum of gold nanocube described throught general dielectric function (TD BEM) and Drude-Lorentz model, the dashed lines represent the pulse frequencies employed in the dynamics of figure 7 .

$$
\vec{E}(t)=E_{0} e^{\left(-\frac{\left(t-t_{0}\right)^{2}}{2 \sigma^{2}}\right)} \sin \left(\omega_{e x c} t\right)
$$

where $\sigma=1.2 \mathrm{fs}$, and its central frequency $\omega_{\text {exc }}$ is close to the dipolar plasmonic frequency (i.e., $\omega_{p}=2.3 \mathrm{eV}$, as obtained through simulation of the absorption spectrum). We calculate the local field at the distance of $3 \AA$ from one of the NP corners employing the TD BEM approach with the general dielectric function and the Drude-Lorentz dielectric function. In dye-functionalized NP setups, $3 \AA$ is a realistic distance for NP localized surface plasmons to be effective at inducing molecular polarization. The absorption spectra of the gold nanocube obtained employing the TD BEM approach with the general dielectric function and the Drude-Lorentz dielectric function are shown in figure 6.

The local fields calculated with general and Drude-Lorentz dielectric function and the incident electric fields are shown in figure 7 with their respective FT, when the system is excited with a frequency lower $\left(\omega_{\text {exc }}=2.07 \mathrm{eV}\right.$, panel $(\mathrm{a})$ and $\left.(\mathrm{b})\right)$ and higher $\left(\omega_{\text {exc }}=2.50\right.$ $\mathrm{eV}$, panel (c) and (d)) than the dipolar plasmonic frequency at $2.3 \mathrm{eV}$. In both cases, with the general dielectric function, the local field propagates in time with a little delay with 

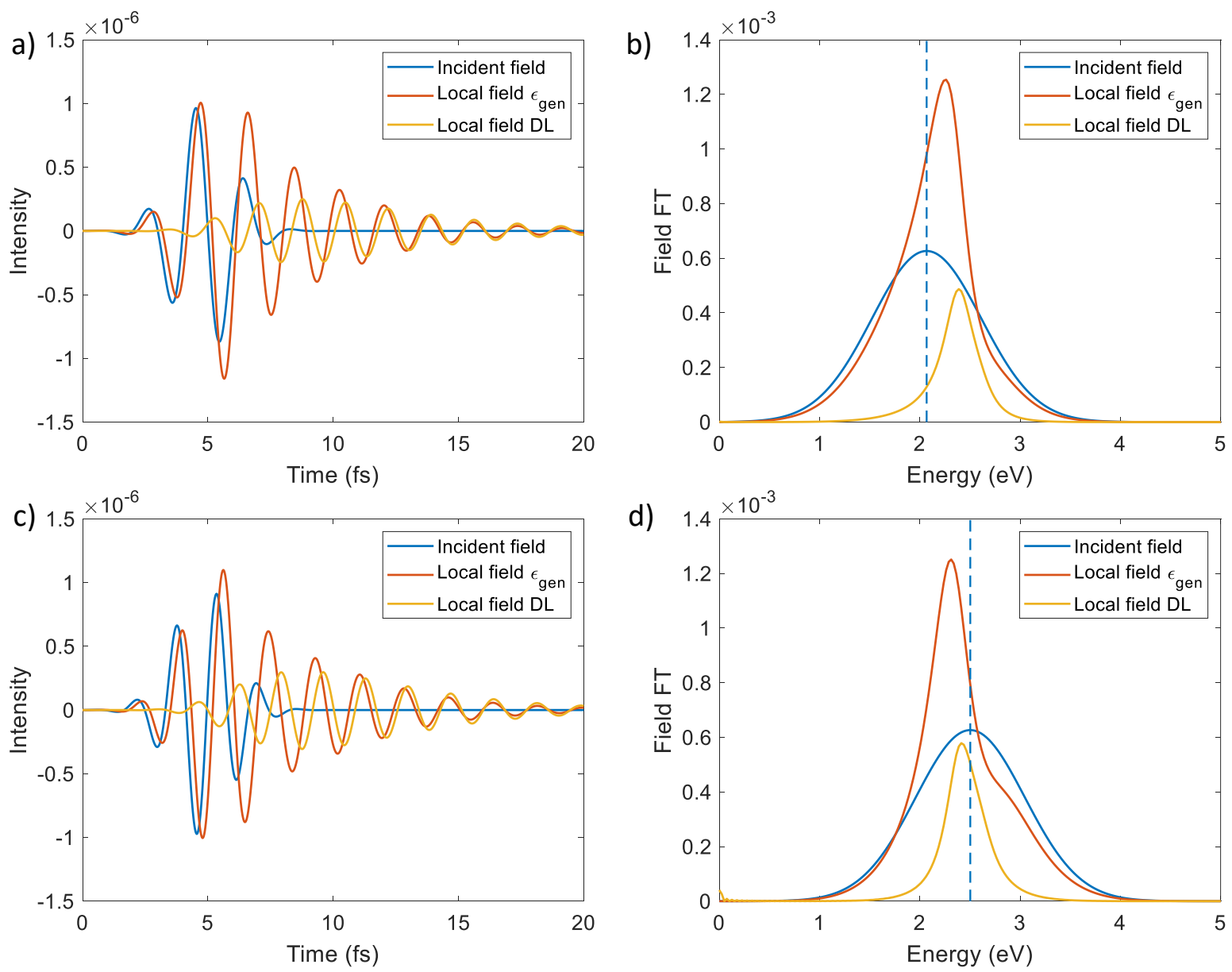

FIG. 7. Time propagation of incident field and local field induced on a gold nanocube at distance 3 $\AA$ when a) $\omega_{e x c}=2.07 \mathrm{eV}$, c) $\omega_{e x c}=2.50 \mathrm{eV}$. b)FT of incident electric field and local field in panel (a), d)FT of incident electric field and local field in panel (c), the dashed lines in panel (b) and (d) represent the pulse frequencies.

respect to the incident field and oscillates with a frequency close to the plasmonic frequency. The local field generated when the dielectric function is modelled as the Drude-Lorentz equation is less intense than the local field generated when the propagation is performed with the general dielectric function. In fact, the DL absorption band is much narrower than the absorption region for the empirical dielectric function, and thus less of the incident field (that spans a range of frequencies) is absorbed and translated in a local field.

Notice that the local fields decay in time with a rate equal to $\gamma_{p}$. We evidence such an exponential decay with a purple line in 7a and c. Remarkably, the field's main frequency shifts from $\omega_{\text {exc }}$ to $\omega_{p}$ as a consequence of the polarization induced on the NP surface. 
Moreover, the FT of the local fields show an inhomogeneous broadening corresponding to the frequencies of the excitation band when the general dielectric function is employed, while with Drude-Lorentz dielectric function only one frequency can be excited and the local field cannot be influenced by the presence of interband transitions. When the central excitation energy is larger than the plasmonic frequency (panel (c) and (d) of figure 7) a larger portion of the polarizability spectrum is superposed to the electric field FT, due to a more pronounced shoulder at $2.7 \mathrm{eV}$. These results point to the connection between the real part of the complex pole frequency and the leading frequency of the local electric field, as well as the connection between the imaginary part of such pole frequency and the decay rate in the time-dependent profile. As anticipated earlier, such a connection might be extremely useful to control the excitation of specific states of a molecule close to the NP.

\section{G. Excitation of a molecule close to a nanocube}

In this section, we report an example of real-time propagation of the electronic wavefunction of a molecule subject to an external electromagnetic field and close to a gold nanocube. In particular, we model the NP dielectric function via Drude-Lorentz equation and via the empirical, frequency dependent dielectric function, to show how such different descriptions affect the results of the dynamics. To this aim, we consider an LiCN molecule at distance $5 \AA$ from one corner of a gold nanocube, with its principal axis placed along the direction of the nanocube diagonal, as in panel (a) of figure 8 . The nanoparticle surface has been discretized in 764 tesserae with different areas, refining the region close to the molecule.

The system interacts with a gaussian enveloped sinusoidal electric field with $\sigma=1.21$ fs and centred at $t_{0}=4.84 \mathrm{fs}$ (see equation 31 ). We perform the dynamics of the system using two different pulse frequencies: $2.40 \mathrm{eV}$ (corresponding to the lowest plasmonic frequency) and $5.92 \mathrm{eV}$ (corresponding to the highest plasmonic frequency). Panel (b) of figure 8 shows the absorption spectra of the gold nanocube described with the Drude-Lorentz dielectric function and the empirical dielectric function, the excitation frequencies are reported as dashed lines. As in the previous cases (panel (c) of figure 1) the Drude-Lorentz dielectric function can only partially describes the absorption spectrum of gold nanoparticles, indeed only one excitation frequency (in this case at $2.40 \mathrm{eV}$ ) contributes to the NP spectrum.

Panel (c) and (d) of figure 8 show the dynamics of the LiCN first excited state popu- 
a)
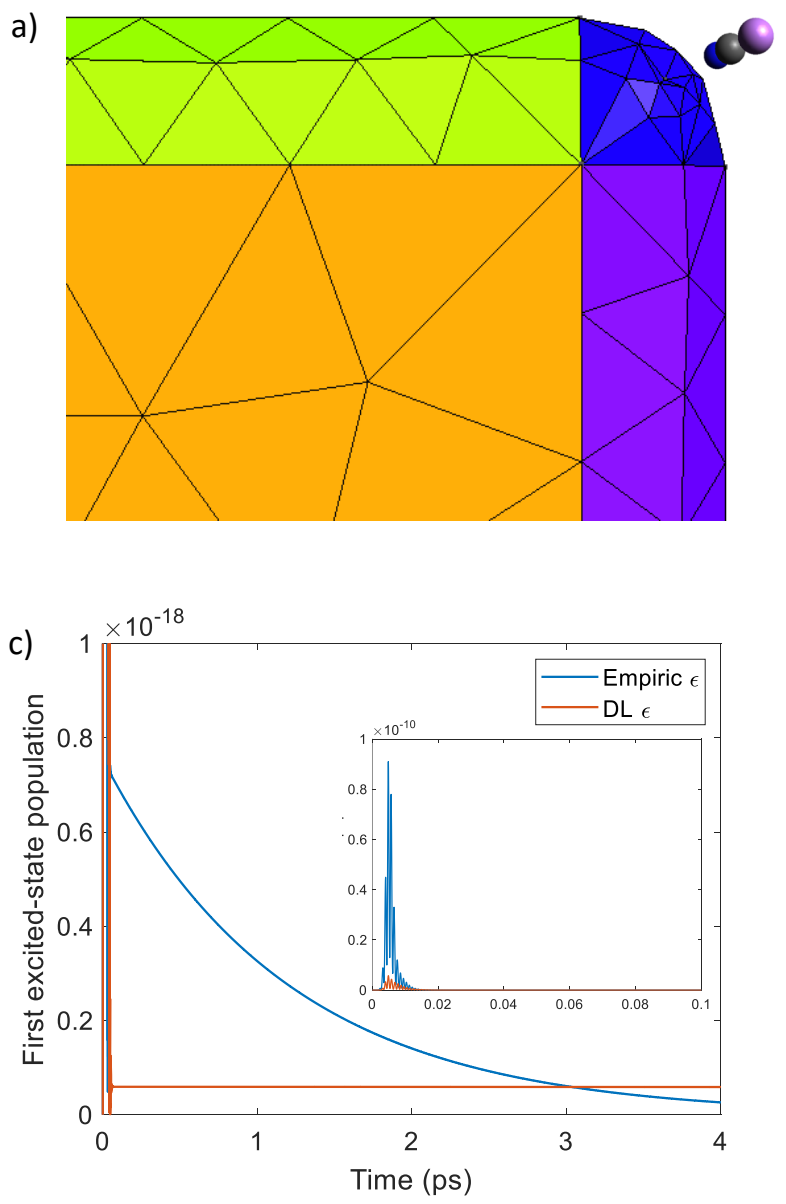
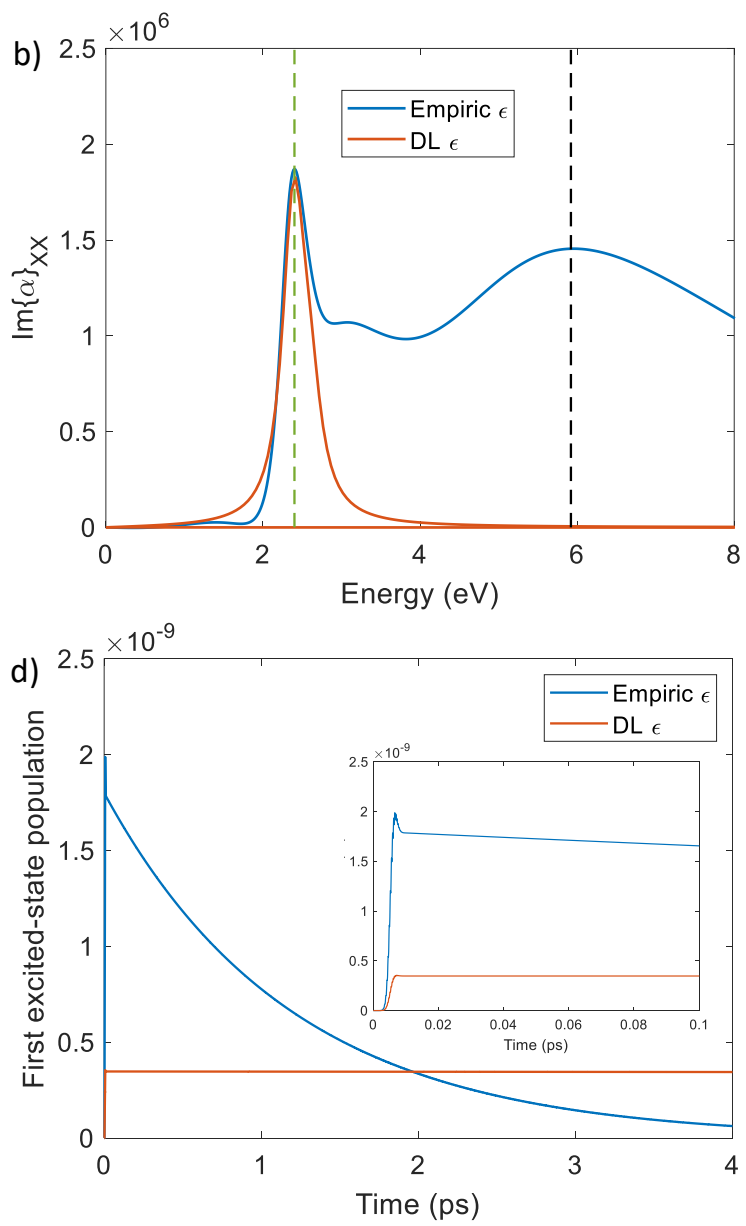

FIG. 8. a) Representation of the gold nanocube corner tessellation and LiCN molecule, b) absorption spectrum of gold nanocube when using its empirical dielectric function and Drude-Lorentz dielectric function. Time evolution of the first excited state population of LiCN molecule at distance $5 \AA$ from the corner of a gold nanocube describing the NP through its empirical dielectric function and the Drude-Lorentz dielectric function when interacting with a gaussian enveloped sinusoidal electric field with pulse frequency $2.40 \mathrm{eV}$ (c) and $5.92 \mathrm{eV}$ (d). The insets are magnifications of the first few tens of fs of the evolution.

lation (neglecting spontaneous emission) when the pulse frequency is $2.40 \mathrm{eV}$ and $5.92 \mathrm{eV}$, respectively. The $2.40 \mathrm{eV}$ pulse frequency (green line in panel (b) of figure 8) allows to excite the plasmonic peak, but the LiCN first excited state is only transiently populated at the beginning of the dynamics (inset of panel(c)) because it is out of resonance (excitation energy is $6.34 \mathrm{eV}$ ). The final population after the pulse is therefore very small, both for the TD BEM with the empirical dielectric function and with the Drude-Lorentz one. Yet, 
such populations are very different one from the other. In fact, the nanoparticle dielectric response at higher energies is (slightly) excited only using TD BEM with empirical dielectric function, and not in the Drude-Lorentz case. The presence of the interaction with the NP in the empirical dielectric function case is exhibited also by the decay of the excited state population, that depends on the possibility to transfer energy from the molecule to the NP.

The population of LiCN first excited is higher when the frequency pulse is closer to the excitation energy of the molecule (panel (d) of figure 8), as expected. Nevertheless, when the dielectric function of the NP is modelled as the Drude-Lorentz equation a smaller population is excited than when the empirical dielectric function is used. In the Drude-Lorentz case the molecule is almost completely excited by the incident field because the local field generated by surface plasmons is not resonant with LiCN excitation energy. On the other hand, in the TD BEM case, the local field generated close to the NP surface, due to the interaction with the incident field, contributes to the excitation of the molecule. Moreover in the latter case the population decays in time because of the energy transfer from the molecule to the NP, as in panel (c).

In summary, the different models of the NP dielectric function differ both for the initial population of an excited state and the subsequent evolution of the population as a function of time, showing clearly the importance of using the realistic empirical dielectric function rather than the model Drude Lorentz one.

\section{CONCLUSION}

In this work, we developed a novel time-dependent Boundary Element Method (TD BEM) suitable to study the real-time dynamics of plasmonic NPs of arbitrary geometrical shape and material. The strong point of our approach lies in the consideration of a general frequency dependence for the NP dielectric function, possibly coming from measurements or numerical calculations, keeping the favorable small number of finite elements typical of BEM approaches. In practice, we successfully fit an auxiliary function depending on the frequency-dependent complex dielectric function -known by points- to an expansion in Drude-Lorentz (DL) terms. Such a DL expansion is convenient to obtain our TD BEM

from a FT of the more customary BEM formulation in the frequency domain (Freq BEM), without further approximations. At the core of our approach, there are equations of motion 
(EOM) governing the evolution of polarization charges induced at the surface of the NP by an external electric field. There is one EOM per each DL term in the aforementioned expansion. Such EOM are second-order differential equations resembling those of a damped and forced harmonic oscillators, and they couple the evolution of both (a) distant point-like polarization charges and (b) polarization charges contributions associated with different DL terms.

As anticipated, the main goal of TD BEM is to provide insights on plasmonic resonances evolving in real time, particularly in view of coupling with a microscopic time-dependent description of a molecule or anyway an external charge distribution. In this article we establish that TD BEM shows little departure from Freq BEM results for any of the NPs we have studied, independently of their geometry and underlying material. Moreover, we show that our TD BEM implementation is numerically robust by comparing with analytical benchmark results for spheres and ellipsoids. Remarkably, arriving to such a good agreement requires a careful selection of (i) the tessellation resolution and (ii) the time step in the propagation algorithm for the polarization charges. Furthermore, we discuss some properties of the plasmonic near field that allow to tune the spectral shape of light pulses in the proximity of a NP. Generally speaking, BEM approaches have been shown to be particularly suitable to be interfaced with first/principle descriptions of molecules, independently from the underlying basis sets (e.g., localized,[26] or grid based[41]). Therefore, the present approach opens the way to a realistic, flexible and computationally effective time-dependent description of the electronic dynamics of molecules close to metal nanoparticles (including a solvent medium), able to describe many aspects of molecular nanoplasmonics.

\section{SUPPLEMENTARY MATERIAL}

We report in supplementary material: plot and data for fitting of gold $f(\omega)$ function with four and five DL terms (Fig. S1, Tab. S1 and S2); plot for fitting of silver $f(\omega)$ function with three, four and five DL terms (Fig. S2) and data for fitting of silver $f(\omega)$ function with three, four, five and six DL terms (Tab. S3, S4, S5 and S6); plot for fitting of rhodium $f(\omega)$ function with three, four and five DL terms (Fig. S3) and data for fitting of silver $f(\omega)$ function with three, four, five and six DL terms (Tab. S7, S8, S9 and S10). We provide an analysis of the role of the time-step in the EOM numerical propagation of TD BEM (fig 
S14). CIS energies of $\mathrm{LiCN}$ are reported in table S11.

\section{ACKNOWLEDGMENTS}

We thank the members of the 'Nanostructures and (bio)molecules modeling' group from the Department of Chemistry at the University of Padova, and the CNR Institute for Nanosciences at Modena, for stimulating discussions. We acknowledge financial support from European Research Council (ERC) under the European Union's Horizon 2020 research and innovation programme, through the project TAME-Plasmons (Grant agreement No. 681285). Computational work has been partially carried out on the C3P (Computational

Chemistry Community in Padua) HPC facility of the Department of Chemical Sciences of the University of Padua. Additionally, G.G. acknowledges support from the Abdus Salam International Centre for Theoretical Physics (ICTP) through the grant NT09-OEA.

\section{DATA AVAILABILITY}

The data that supports the findings of this study are available as supplementary material.

[1] S.-Y. Ding, J. Yi, J.-F. Li, B. Ren, D.-Y. Wu, R. Panneerselvam, and Z.-Q. Tian, Nature Reviews Materials 1, 1 (2016).

[2] R. P. Van Duyne, Science 306, 985 (2004).

[3] V. Giannini, A. I. Fernández-Domínguez, S. C. Heck, and S. A. Maier, Chemical Reviews 111, 3888 (2011).

[4] L. Nair and C. Laurencin, J. Biomed. Nanotechnol. 3, 301 (2007).

[5] F. Le, D. Brandl, Y. Urzhumov, H. Wang, J. Kundu, N. Halas, J. Aizpurua, and P. Nordlander, ACS Nano 2, 707-718 (2008).

[6] S. Kim, J. Jin, Y.-J. Kim, I.-Y. Park, Y. Kim, and S.-W. Kim, Nature 453, 757-760 (2008).

[7] O. Andreussi, A. Biancardi, S. Corni, and B. Mennucci, Nano Lett. 13, 4475-4484 (2013).

[8] J. Lee, J. Song, D. Lee, and Y. Pang, Scientific Reports 9 (2019).

[9] W. Zhu, R. Esteban, A. G. Borisov, J. J. Baumberg, P. Nordlander, H. J. Lezec, J. Aizpurua, and K. B. Crozier, Nature Communications 7, 11495 (2016). 
[10] C. Ciracì, R. T. Hill, J. J. Mock, Y. Urzhumov, a. I. Fernández-Domínguez, S. a. Maier, J. B. Pendry, a. Chilkoti, and D. R. Smith, Science (New York, N.Y.) 337, 1072 (2012).

[11] U. U. Hohenester, Nano and quantum optics : an introduction to basic principles and theory (Springer, 2020) p. 665.

[12] J. L. Volakis and K. Sertel, Integral Equation Methods for Electromagnetics (2012) pp. 1-392.

[13] R. Fuchs, Physical Review B 11, 1732 (1975).

[14] F. García de Abajo and A. Howie, Physical Review B 65, 115418 (2002).

[15] U. Hohenester and A. Trügler, Computer Physics Communications 183, 370 (2012), arXiv:1109.5783.

[16] M. T. Reid and S. G. Johnson, IEEE Transactions on Antennas and Propagation 63, 3588 (2015), arXiv:1307.2966.

[17] J. Marcheselli, D. Chateau, F. Lerouge, P. Baldeck, C. Andraud, S. Parola, S. Baroni, S. Corni, M. Garavelli, and I. Rivalta, Journal of chemical theory and computation 16, 3807 (2020).

[18] X.-W. Chen, A. Mohammadi, A. H. B. Ghasemi, and M. Agio, Molecular Physics 111, 3003 (2013).

[19] E. Wientjes, J. Renger, A. G. Curto, R. Cogdell, and N. F. van Hulst, Nature communications 5, $4236(2014)$.

[20] J. Fregoni, G. Granucci, E. Coccia, M. Persico, and S. Corni, Nature Communications 9, 1 (2018).

[21] S. Pipolo and S. Corni, The Journal of Physical Chemistry C 120, 28774 (2016).

[22] S. Corni, S. Pipolo, and R. Cammi, Journal of Physical Chemistry A 119, 5405 (2015).

[23] F. Hao and P. Nordlander, Chemical Physics Letters 446, 115 (2007).

[24] H. S. Sehmi, W. Langbein, and E. A. Muljarov, Phys. Rev. B 95, 115444 (2017).

[25] B. Mennucci and S. Corni, Nature Reviews Chemistry 3, 315 (2019).

[26] J. Tomasi, B. Mennucci, and R. Cammi, Chemical Reviews 105, 2999 (2005).

[27] E. Cancès, B. Mennucci, and J. Tomasi, The Journal of Chemical Physics 107, 3032 (1997).

[28] E. Vanden-Eijnden and G. Ciccotti, Chemical Physics Letters 429, 310 (2006).

[29] S. Mukamel, Principles of nonlinear optical spectroscopy (Oxford University Press, 1995).

[30] P. B. Johnson and R. W. Christy, Phys. Rev. B 6, 4370 (1972).

[31] A. D. Rakić, A. B. Djurišić, J. M. Elazar, and M. L. Majewski, Appl. Opt. 37, 5271 (1998). 
[32] E. Palik, Handbook of Optical Constants of Solids (Academic Press: Cambridge, MA, USA, 1998).

[33] E. Coccia and S. Corni, The Journal of Chemical Physics 151, 044703 (2019).

[34] C. Dykstra, G. Frenking, K. Kim, and G. Scuseria, Theory and applications of computational chemistry: the first forty years (Elsevier, 2011).

[35] M. W. Schmidt, K. K. Baldridge, J. A. Boatz, S. T. Elbert, M. S. Gordon, J. H. Jensen, S. Koseki, N. Matsunaga, K. A. Nguyen, S. Su, et al., Journal of computational chemistry 14, 1347 (1993).

[36] G. Grosso and G. Pastori Parravicini, Solid state physics (Academic Press, 2000) p. 727.

[37] L. Novotny and B. Hecht, Principles of Nano-Optics (Cambridge University Press, Cambridge, UK, 2006).

[38] P. G. Etchegoin, E. Le Ru, and M. Meyer, The Journal of chemical physics 125, 164705 (2006).

[39] M. G. Blaber, A. I. Henry, J. M. Bingham, G. C. Schatz, and R. P. Van Duyne, Journal of Physical Chemistry C 116, 393 (2012).

[40] V. Myroshnychenko, J. Rodríguez-Fernández, I. Pastoriza-Santos, A. M. Funston, C. Novo, P. Mulvaney, L. M. Liz-Marzán, and F. J. García de Abajo, Chem. Soc. Rev. 37, 1792 (2008).

[41] G. Gil, S. Pipolo, A. Delgado, C. A. Rozzi, and S. Corni, Journal of Chemical Theory and Computation 15, 2306 (2019). 